\title{
Orthodontic treatment-related risks and complications: part II periodontal complications
}

\author{
Tiro, Alisa* \\ *Department of Orthodontics, School of Dental Medicine, University of Sarajevo, Bosnia and Herzegovina
}

\begin{abstract}
Introduction: Orthodontic treatment, in addition to patient benefits, includes certain risks and complications that should not be ignored. The side-effects of orthodontic therapy all therapists want to avoid or reduce to a minimum.

The occurrence of these complications depends on the orthodontic technique, medical knowledge in this field, patient's general and oral health, and oral hygiene habits. Part of the risk and complications depend on the patient's ability to understand and cooperate during the therapy. For this reason, it is a medico-legal obligation to inform our patients about side effects of any medical intervention what will be undertaken. In the previous issue, an overview of local complications of orthodontic treatment - dental complications was presented.

The aim: The aim of this part is to present a detailed overview of the most common complications with local effect- periodontal complications in the context of the contemporary attitude of the risks and complications associated with orthodontic treatment.

Material and methods: Classification was presented by Graber, 2004th, in its publication, "Risk Management in Orthodontics: An Expert's Guide to Malpractice," used as a starting point for the development of this review. A complex search was performed (Pub Med, Google Scholar, etc.), with the restriction upon of publication date (beginning with 2004) to find relevant current studies. Search keywords were combinations of words: complication, orthodontic treatment, risks, side effects.

Concluding remarks: Periodontal complications are the most common side effects linked with orthodontic treatment according to contemporary literature. Serious analysis of potential risk for periodontal complications is mandatory for orthodontic patients before the treatment starts. It is the only correct way to minimize complications during and after orthodontic treatment.
\end{abstract}

Orthodontic Treatment-Related Risks and Complications: Part II periodontal complications. South Eur J Orthod Dentofac Res. 2018;5(1):18-20.

Submitted: November 25, 2017; Revised: April 28, 2018; Published: May 08, 2018

\section{INTRODUCTION}

Orthodontic treatment is a complex medical intervention carried out over a long period of time. During this time, risks may turn into complications. It is necessary to identify the risks that are associated with the orthodontic intervention to be applied, as there are numerous complications possibly linked to that treatment.

The occurrence of these complications depends on the orthodontic technique, medical knowledge in this field, patient's general and oral health, and oral hygiene habits. These must be considered even from the start because it might influence the treatment objectives, phases, and goals.

Corresponding Author:

Tiro Alisa

Department of Orthodontics,

School of Dental Medicine, University of Sarajevo,

Bolnička 4a, Sarajevo 71000,

Bosnia and Herzegovina

e-mail:atiro@sf.unsa.ba
It is recommended that each patient makes a serious analysis of the risks and potential complications of treatment, which can be followed by signed informed consent. ${ }^{1}$

The possible risks and complications associated with orthodontic treatment, according to their effects, can be divided into two main groups: the risks and complications of systemic effects and the risks and complications of local effects. ${ }^{2,3}$

The most common risks and complications of orthodontic procedures with local effect are:

1. Periodontal complications

2. Dental complications

3. Soft tissue alterations

4. Temporomandibular joint disorders

5. Unsatisfactory treatment outcomes

The detailed overview one of the most common local complication of orthodontic treatment - dental complications was presented in the previous issue. This part will discuss the risks and complications of local effects as a periodontal complication. 


\section{BACKGROUND}

The classification was presented by Graber (2004) in the publication Risk Management in Orthodontics: Experts' Guide to Malpractice and was used as a starting point for the development of this study. A complex electronic search was performed using Pub Med, and Google Scholar, with the restriction to the year of publication (beginning with 2004), the design of study and language. Inclusion criteria were: year of publication 2004 and further, full text, clinical, epidemiological study, review article, and English language. Exclusion criteria were: paper publishing before 2003, abstracts, conference abstracts, letters to editor and animal studies Search keywords and phrases were combined: complications, orthodontic treatment, risk, systemic, local, and side effects. Dental complications as one of the most common forms of orthodontic treatment side effects are described in part I of this review.

This part will discuss a periodontal complication.

\section{Periodontal complications}

The orthodontic tooth movement is based on an inflammatory reaction in the periodontium. Orthodontic tooth movement is induced by mechanical stimulus and facilitated by the remodeling of the periodontal ligament and alveolar bone. The remodeling activities and the ultimately tooth displacement are the consequence of an inflammatory process. ${ }^{4}$

An important number of inflammatory mediators are produced in sufficient amounts to diffuse into the gingival crevicular fluid and their analysis has been used to evaluate the local cellular metabolism that accompanies bone remodeling process during orthodontic tooth movement. 4,5

Gingival inflammation, discomfort or pain and root resorption are common undesirable side effects of orthodontic treatment. Gingival health is compromised during orthodontic treatment because of alterations in the composition of bacterial plaque and consequently the development of gingivitis, especially when fixed orthodontic appliances are used. ${ }^{6,7}$

Periodontal complications are the most common side effects linked to orthodontics. ${ }^{8}$

The periodontal complications as orthodontic therapy consequences may be present in various forms: gingivitis, periodontitis, gingival recession or hypertrophy, alveolar bone loss, dehiscence, fenestrations, interdentally fold, and dark triangles. ${ }^{9}$

\section{Gingival hyperplasia}

Gingival hyperplasia is a relatively frequent pathologic condition during orthodontic treatment. One of the most common soft tissue problems associated with fixed orthodontic appliances is gingival enlargement or hyperplasia. The prevalence rate of $10 \%$ is reported. ${ }^{10}$ Gingival hyperplasia of inflammatory origin occurs in the 1-2 month of the orthodontic treatment. Starts in the interdentally papillae, and continues as a localized or generalized form. ${ }^{11}$

Oral hygiene can be easily altered in such cases because of additive and irritative effects of orthodontics appliances.
Orthodontic brackets and elastics might induce accumulation of dental plaque. If the oral hygiene is not adequate, it leads to gingivitis. ${ }^{8,12}$

Gingival inflammation around orthodontic bands leads to pseudo pockets, which usually disappears approximately three months after the deboning of the brackets. A greater loss of the clinical attachment level, found distally in the dental arches, has been reported by a few clinical studies. ${ }^{13,14,15}$

This could be the result of poor oral hygiene in the molar regions and the presence of molar bands, which favor the accumulation of food. That is a reason to choose molar tubes instead molar bands. In order to ensure safer medical care, it is a necessary to insist on the importance of maintaining good oral hygiene and the monitoring of the periodontal status. ${ }^{16}$ In some high-risk patients, such as those with diabetes mellitus or epilepsy, it is mandatory to do an examination and dental cleaning at least once every three months. It is also recommended to avoid the use of hooks and elastic ligatures as much as possible, with chains and metallic ligatures being preferable to elastomeric ones. ${ }^{17}$

\section{Gingival recession}

A gingival recession of the incisors is a condition that is affected by several factors: the width of the attachment gingival, the change in the inclination during the treatment, oral hygiene, the brushing technique, the predetermined periodontal condition and the thickness of the symphysis. ${ }^{18}$ Contradictory results of statistically significant correlation between the mentioned factors were found. The low statistical significance is the difference in the amount of recession between proclined and non-proclined incisors. To solve the dilemma of recessional connection and changes in tooth inclination during orthodontic treatment, clinical studies required will take into account the state of hygiene and gingiva before and after treatment. ${ }^{19}$ There is a risk that patients with the pre-existing recession have a chance for a further recession during orthodontic tooth movement. ${ }^{20}$ The risk can be minimized by kept proper oral hygiene, used nontraumatic brushing technique. It is patient-related factor. The therapist related factors including closely monitoring of the recession during treatment to ensure that is not progressing. ${ }^{21,22}$

\section{Alveolar bone loss}

Alveolar bone support is important for periodontal health; specifically, it is essential to the stability of anterior teeth and thus to acceptable esthetics. The mandibular symphysis is the anatomic structure that limits the movement of the lower incisors, and the bony thickness of the lower anterior teeth is thin and susceptible to periodontal disease. ${ }^{23}$

In patients with mandibular prognathism, the occurrence of alveolar bone loss or fenestration is more common in the lower anterior teeth. Some studies have shown that more proclined teeth compared with less proclined teeth or untreated teeth might be associated with a higher tendency to develop alveolar bone loss. ${ }^{24}$ But the other study did not report alveolar bone change around the mandibular incisors before and after the surgical orthodontic treatment. ${ }^{20,25}$ 


\section{Dark triangles}

This is a condition much more influenced by the patient-related factors than therapist related factors. The height of alveolar bone relative to interproximal contact is a significant factor in determining where a papilla fills the gingival embrasure. The shape of the teeth plays a critical role in the presence of dark triangles. ${ }^{26}$ The location and size of interproximal contact and divergent tooth angulation have been cited as a potential cause of open gingival embrasures. According to current literature, the dark triangles between central maxillary incisors and cervical margin of gingiva are directly consequence of the orthodontic treatment. Adult patients present a risk because they often have some dental conditions that may complicate treatment such as tooth wear, poorly contoured restorations, and periodontal disease. ${ }^{27,28}$

\section{CONCLUDING REMARKS}

Successful risk management of periodontal complications is one of the most important factors that influence the objectives and outcome. For the most periodontal complications, there is not enough evidence that they are directly caused by orthodontic treatment. The patient's previous periodontal condition is the primary factor of periodontal complications during and after orthodontic treatment. The orthodontic procedures should provide maximum benefit with minimal side effects. To ensure safer medical care, orthodontic treatment, as a part of dentalmedical science, must be performed with great precaution.

\section{CONFLICT OF INTEREST}

The author declare no conflict of interest.

\section{REFERENCES}

1. Graber T, Eliades T, Athanasius AE. Risk Management in Orthodontics: Experts Guide to Malpractice, 2004. Quintessence Publishing Co, Inc, Chicago.

2. Lau PY. Wong RWK. Risks and complications in orthodontic treatment. Hong Kong Dent J. 2006;3(1):15-22.

3. Ackerman M. Evidence-based orthodontics for the 21st century. J Am Dent Assoc. 2004;135(2):162-7.

4. Bletsa A, Berggreen E, Brudvik P. Interleukin-1 $\alpha$ and tumor necrosis factor- $\alpha$ expression during the early phases of orthodontic tooth movement in rats. Eur J Oral Sci. 2006;114(5):423-429.

5. Dannan A. An update on periodontic-orthodontic interrelationships. J Indian Soc Periodontol. 2010;14(1):66-71.

6. Samah Alfuriji, Nora Alhazmi, Nasir Alhamlan, et al.The Effect of Orthodontic Therapy on Periodontal Health: A Review of the Literature, Int J Dent Oral Haelth. 2014;(4):58504.

7. Krishnan V, Ambili R, Davidovitch Z, Murphy NC. Gingiva and Orthodontic Treatment. Sem in Orthodontics, 2007;13(4):257-271.

8. Kouraki E, Bissada NF, Palomo JM, Ficara AJ. Gingival enlargement and resolution during and after orthodontic treatment. N Y State Dent J. 2005; 71(4):34-7.

9. Bollen AM, Cunha-Cruz J, Bakko DW, Huang GJ, Hujoel PP. The effects of orthodontic therapy on periodontal health: a systematic review of controlled evidence. J Am Dent Assoc. 2008;139(4):413-22.

10. Preoteasa CT, Ionescu E, Preoteasa E . Risks and Complications Associated with Orthodontic Treatment. chapter 18. in Bourzgui F. Orthodontics Basic Aspects and Clinical Considerations, 2012. InTec. p 420.

11. Ristic M, Vlahovic-Svaibc M, Sasic M, Zelic O. Clinical and microbiological effects of fixed orthodontic appliances on periodontal tissue in adolescents. Orthod Craniofac Res.2007;10(4):187-195.

12. Vizitiu TC, Ionescu E. Microbiological changes in orthodontically treated patients. Therapeutics. J Pharmacol Clin Toxicol. 2010;14(4):283-6.

13. Nakaš E, Džemidžić V, Tiro A, Pasić E, Hadžić S. Antimicrobial activity of chlorhexidine in patients with fixed orthodontic appliances. Braz J Oral Sci. 2011;10(2):79-82.

14. Freitas AO, Marquezan M, Nojima Mda C, Alviano DS, Maia LC. The influence of orthodontic fixed appliances on the oral microbiota: a systematic review. Dental Press J Orthod. 2014;19(2):46-55.

15. Klukowska M, Bader A, Erbe C, Bellamy P, White DJ, Anastasia MK, Wehrbein H. Plaque levels of patients with fixed orthodontic appliances measured by digital plaque image analysis. Am J Orthod Dentofacial Orthop. 2011;139(5):e463-70.

16. Kerosuo HM, Dahl JE. Adverse patient reactions during orthodontic treatment with fixed appliances. Am J Orthod Dentofacial Orthop. 2007;132(6):789-95.

17. Meeran NA. Iatrogenic possibilities of orthodontic treatment and modalities of prevention. J Orthod Sci. 2013;2(3):73-86.

18. Shah AA, Sandler J. Limiting factors in orthodontic treatment: 1 . Factors related to patient, operator and orthodontic appliances. Dent Update. 2006;33(1):43-4, 46-8,51-2.

19. Yared KF, Zenobio EG, Pacheco W. Periodontal status of mandibular central incisors after orthodontic proclination in adults. Am J Orthod Dentofacial Orthop. 2006;130(1):6.e1-8.

20. Kim Y, Park JU, Kook YA. Alveolar bone loss around incisors in surgical skeletal Class III patients. Angle Orthod. 2009;79(4):676-82.

21. Tyndall, D. A. and S. Rathore. Cone-beam CT diagnostic applications: caries, periodontal bone assessment, and endodontic applications. Dent Clin North Am. 2008;52(4):825-41.

22. Ionescu E, Teodorescu E, Badarau A, Grigore R, Popa M. Prevention perspective in orthodontics and dento-facial orthopedics. J Med Life. 2008;1(4):397-402.

23. Joss-Vassalli I, Grebenstein C, Topouzelis N, Sculean A, Katsaros C. Orthodontic therapy and gingival recession: a systematic review. Orthod Craniofac Res. 2010;13(3):127-41.

24. Kyung-Min Lee, Yong-Il Kim, Soo-Byung Park, and Woo-Sung Son. Alveolar bone loss around lower incisors during surgical orthodontic treatment in mandibular prognathism. The Angle Orthod. 2012;82(4):637-644.

25. Leung CC, Palomo L, Griffith R, Hans MG. Accuracy and reliability of cone-beam computed tomography for measuring alveolar bone height and detecting bony dehi-scences and fenestrations. Am J Orthod Dentofacial Orthop. 2010;137(4):109-19.

26. Crescini A, Nieri M, Buti J, Baccett T, Prato GP. Orthodontic and periodontal outcomes of treated impacted maxillary canines: An appraisal of prognostic factors. Angle Orthod.2007;77(4): 571-7.

27. Genco RJ, Borgnakke SW. Risk factors for periodontal disease. Periodontol. 2000;62(1):59-94.

28. van Gastel J, Quirynen M, Teughels W, Carels C. The relationships between malocclusion, fixed orthodontic appliances and periodontal disease. A review of the literature. Aust Orthod J. 2007;23(2):121-9. 